\title{
A note on the dynamics of an HIV infection model using Padé approximants
}

\author{
PEDRO PABLO CARDENAS ALZATE \\ Department of Mathematics and GEDNOL \\ Universidad Tecnológica de Pereira \\ Pereira, COLOMBIA
}

\author{
JOSE RODRIGO GONZALEZ GRANADA \\ Department of Mathematics and GEDNOL \\ Universidad Tecnológica de Pereira \\ Pereira, COLOMBIA
}

Abstract: In this work, we use Padé's approaches in solving a system of ordinary nonlinear differential equations which arises in the model for HIV infection of $C D 4^{+} T$ cells. Some graphs are presented to show the reliability and simplicity of this method as well as the algorithms implemented in the analysis of the model.

Key-Words: Padé approximants, Model for HIV of $C D 4^{+} T$ cells, Semi-Analytical Method, Normal Padé Table.

Received: March 13, 2020. Revised: August 18, 2020. Accepted: August 30, 2020. Published: September 25, 2020.

\section{Introduction}

The non-linear problems form a major line of research in the sciences in general since many phenomena are modeled using nonlinear equations. It is also true that in most cases, it is not possible to find analytical solutions to such models and therefore knowledge of efficient numerical methods to approximate them is essential. Thus, there are several semi-analytical methods that allow us to approximate the solutions numerically, such as the ADM, the DTM and the Padé approximant method [1].

For this reason, the Padé method is used in the resolution of non-linear problems due to its excellent convergence. This method has proven to be very useful in obtaining quantitative information about the solution of many interesting problems in sciences. The applications of Padé approximants are divided into two classes:

- The provision of efficient rational approaches to special mathematical functions.

- The acquisition of quantitative information about a function.

We can say then that the Padé approaches are the basis of several non-linear techniques which have a connection with the well known $\epsilon$ algorithm. In the specialized literature, several methods are known to find the Padé approximations, so the fundamental objective of this work is to pay special attention to this type of fractions in which the method is applicable presenting the most relevant properties in the applications.

Now, by way of introduction, it is important to note that HIV is a retrovirus that targets the $C D 4^{+} T$ lymphocytes, which are the most abundant white blood cells of the immune system. Although HIV infects other cells also, it wreaks the most havoc on the $C D 4^{+} T$ cells by causing their decline and destruction, thus decreasing the resistance of the immune system.

\section{Padé approximant mehod}

From elemental analysis, divergence in a series of powers is essential to talk about the presence of singularities. This type of divergence shows the problem that polynomials have to approach a function around that singularity, where the basic idea is to represent any function $f(z)$ by a convergent expression [2]. One of the most used techniques that requires as input a finite number of terms is the approximation of Padé. In this method proposed by Padé, this series [13]

$$
f(z)=\sum_{i=0}^{\infty} c_{i} z_{i}
$$

is replaced by a succession of rational functions of the form:

$$
R_{N}^{M}(z)=\frac{\sum_{i=0}^{M} a_{i} z_{i}}{\sum_{i=0}^{N} b_{i} z_{i}} .
$$

Therefore, we use the standardization $b_{0}=1$ and the remaining $M+N+1$ coefficients $a_{0}, \ldots, a_{M}$ and $b_{1}, \ldots, b_{N}$ in Eq.(2) are chosen such that $M+N+1$ 
is the coefficient in the power series expansion (see Eq.(1)). Thay is,

$$
f(z)-R_{N}^{M}(z)=\mathcal{O}\left(Z^{M+N+1}\right),
$$

then

$$
f(z) \sum_{i=0}^{M} b_{i} z_{i}-\sum_{i=0}^{M} a_{i} z_{i}=\mathcal{O}\left(Z^{M+N+1}\right) .
$$

If $\sum a_{i} z_{i}$ is a series representation of $f(z)$, then many cases $R_{N}^{M}(z) \rightarrow f(z)$ when $M, N \rightarrow \infty$, even if $\sum a_{i} z_{i}$ is divergent. Generally, we consider the convergent succession $R_{0}^{J}, R_{0}^{J+1}, \ldots$, where $M=N+J$ with $J$ fixed and $N \rightarrow \infty$.

Therefore, we get

- Case 1: $M \geq N$. Developing the expression (4) we have:

$$
\begin{aligned}
& c_{0}+c_{1} z+c_{2} z^{2}+\ldots+\quad c_{N} z^{N}+\ldots+\quad c_{M} z^{M}+\ldots+\quad c_{M+N} z^{M+N} \\
& +c_{0} b_{1} z+c_{1} b_{1} z^{2}+\ldots+c_{N-1} b_{1} z^{N}+\ldots+c_{M-1} b_{1} z^{M}+\ldots+c_{M+N-1} b_{1} z^{M+N} \\
& +c_{0} b_{2} z^{2}+\ldots+c_{N-2} b_{2} z^{N}+\ldots+c_{M-2} b_{2} z^{M}+\ldots+c_{M+N-2} b_{2} z^{M+N} \\
& +c_{0} b_{N} z^{N}+\ldots+c_{M-N} b_{N} z^{M}+\ldots+c_{M} b_{N} z^{M+N} \\
& =a_{0}+a_{1} z+a_{2} z^{2}+\ldots+a_{N} z^{N}+\ldots+\quad a_{M} z^{M}
\end{aligned}
$$

Then, equating coefficients we obtain:

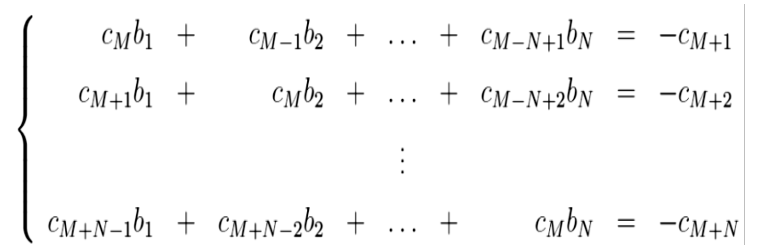

and

$$
\left\{\begin{array}{l}
a_{0}=c_{0} \\
a_{1}=c_{1}+c_{0} b_{1} \\
a_{2}=c_{2}+c_{1} b_{1}+c_{0} b_{2} \\
\vdots \\
a_{N}=c_{N}+c_{N-1} b_{1}+c_{N-2} b_{2}+\ldots+\quad c_{0} b_{N} \\
\vdots \\
a_{M}=c_{M}+c_{M-1} b_{1}+c_{M-2} b_{2}+\ldots+c_{M-N} b_{N}
\end{array}\right.
$$

- Case 2: $M<N$.

In this case and after matching coefficients we get:

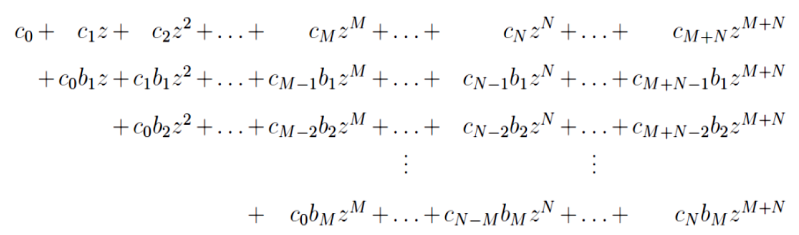

Now, solving these systems we get:

where $\mathcal{A}$ is a matrix $n \times n$ whose entries are given by $A_{i j}=c_{M+i-j}$ with $a_{k}=0$ if $k<0$. Therefore, the coefficients $a_{0}, a_{1} \ldots$, are determined by using Eq.(5)

$$
a_{j}=\sum_{k=0}^{j} c_{j-k} b_{k}, \quad 0 \leq j \leq M,
$$

where $b_{k}=0$ for $k>N$. Here, the resulting rational function $R_{N}^{M}(z)$ is called a Padé approximation.

\subsection{Modified algorithm}

Semi-analytical techniques to solve nonlinear models require an initial vision to determine the solutions as well as the calculation of one or more parameters that fit the initial system. When the parameters are chosen correctly, the results can be very precise, but it is important to emphasize that there is no method for initially choosing this choice. In this work, we suggest to use directly the serial solution for a nonlinear model to find Padé's approximation with highly efficient results $[3,4]$. 
de donde,

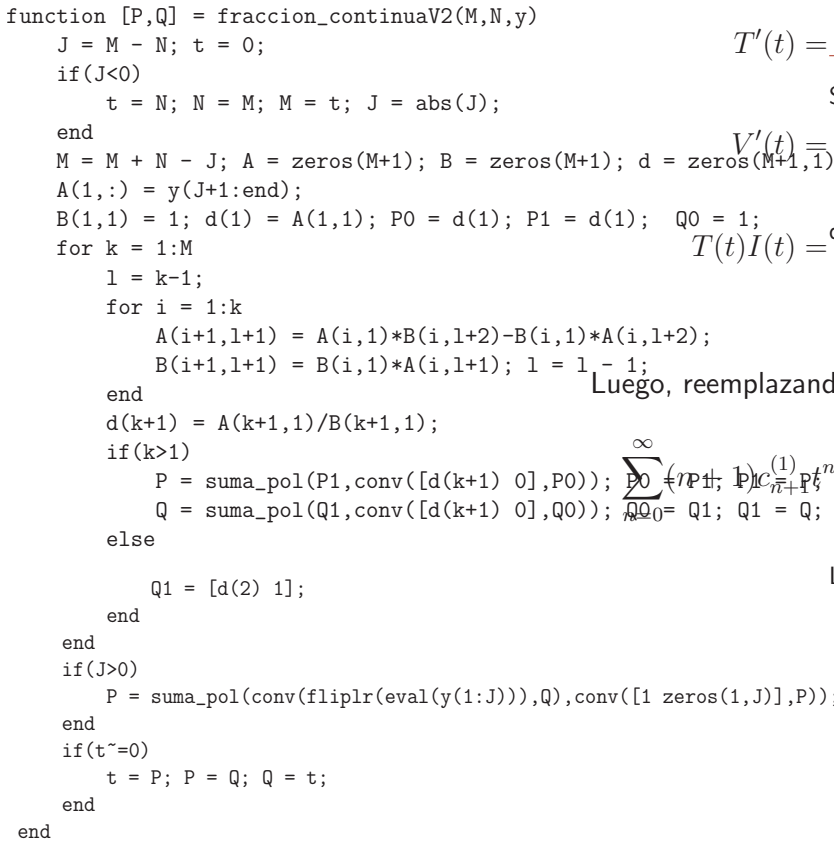

Algorithm 1. Modified algorithm.
Y por tanto, de (4.26)! ? por tanto, de (4.26):

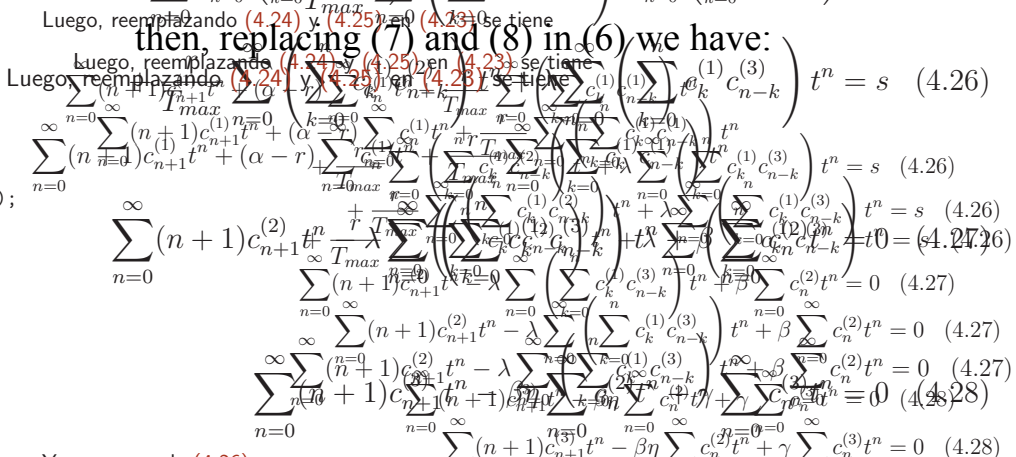

(7) $(4(24: 25)$

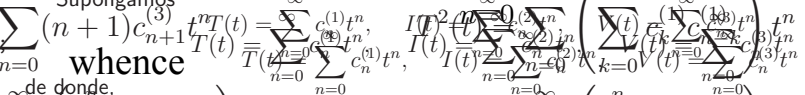
$(44295)$

The method receives as input parameters, in addi- Para por tanto, de (4.26):

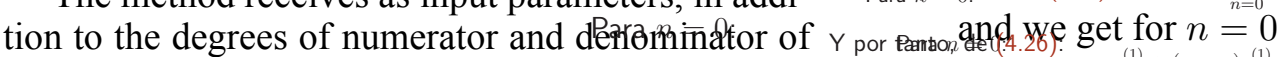
$\sum_{n=0}^{\infty}(n+1) c_{n+1}^{n_{3}} t^{n}-\beta \eta \sum_{n=0}^{\infty} c_{n}^{(2)} t^{n}+\gamma=0$
$\sum_{n=0}^{\infty}(n+1) c_{n+1}^{\infty} t^{n}-\beta \eta \sum_{n=0}^{\infty} c_{n}^{(2)} t^{n}+\gamma \sum_{n=0}^{(3)} c_{n}^{n} c_{n}^{n}=0$ the Padé approximation, the coefficients of the series expansion instead of the function explicitly $[5,6]$.

\section{Dynamics of an HIV infection model}

Below we analyze the dynamics for a model of HIV infection from $C D 4^{+} T$ cells. The components of this

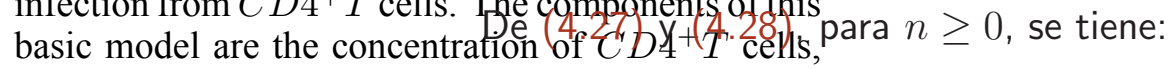
$C D 4^{+} T$ cells infected with HIV and the HIV-free particles, which we will denote by $T(t), I(t)$ and $V(t)$ respectively [7]. These amounts satisfy the following relationship:

$$
(n+1) c_{n+1}^{(2)}-\lambda \sum_{k=0}^{n} c_{k}^{(1)} c_{n-k}^{(3)}+\beta c_{n}^{(2)}=0
$$

$$
(n+1) c_{n+1}^{(3)}-\beta \eta c_{n}^{(2)}+\gamma c_{n}^{(3)}=0
$$

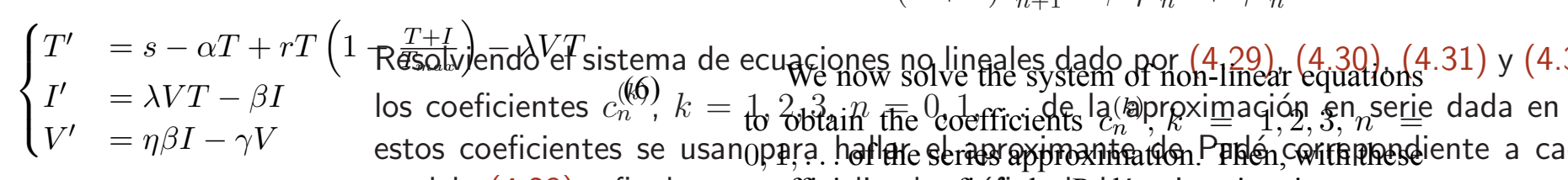

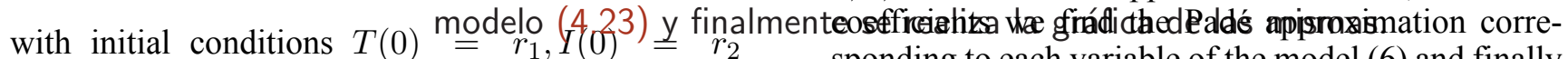
and $V(0)=r_{3}$. In this case, we use the values $s=0.1, \alpha=0.02, \beta=0.3, r=3, \gamma=2.4, \lambda=$ $0.0027, T_{\max }=1500, \eta=10, r_{1}=0.1, r_{2}=0$ and $r_{3}=0.1$.

Now, we assume

clear all; global $\mathrm{N}$ sponding to each variable of the model (6) and finally we make the corresponding graph.

The procedure proposed in this work [8, 9]. , compared to the dan, is much simpler from the algebraic point of view,

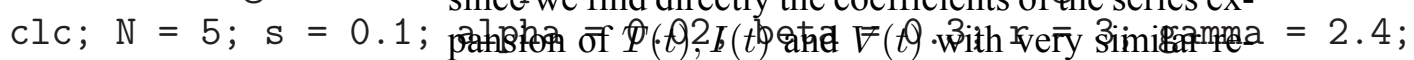

$$
T(t)=\sum_{n=0}^{\infty} c_{n}^{(1)} t^{n}
$$
lambda $=0.0027 ; \mathrm{Tm}=$ sults.00; eta $=10 ; \mathrm{r} 1=0.1 ; \mathrm{r} 2=0 ; \mathrm{r} 3=0.1$;

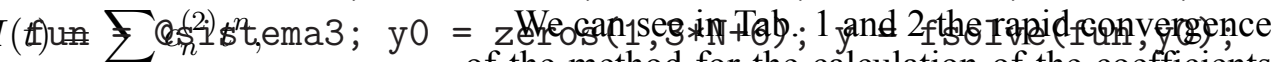
for $n_{k=0}: \mathrm{N}+1$ of the method for the calculation of the coefficients

$$
\begin{aligned}
& \mathrm{ct}(\mathrm{k}+1)=\mathrm{y}(3 * \mathrm{k}+1) ; \\
& \mathrm{ci}(\mathrm{k}+1)=\mathrm{y}(3 * \mathrm{k}+2) ; \\
& \mathrm{cv}(\mathrm{k}+1)=\mathrm{y}(3 * \mathrm{k}+3) ;
\end{aligned}
$$

end

$$
\begin{aligned}
& {[\mathrm{Pt}, \mathrm{Qt}]=\operatorname{pade} 3\left(\mathrm{ct}^{454}, 3,3\right)} \\
& {[\mathrm{Pi}, \mathrm{Qi}]=\operatorname{pade} 3(\mathrm{ci}, 3,3) ;}
\end{aligned}
$$




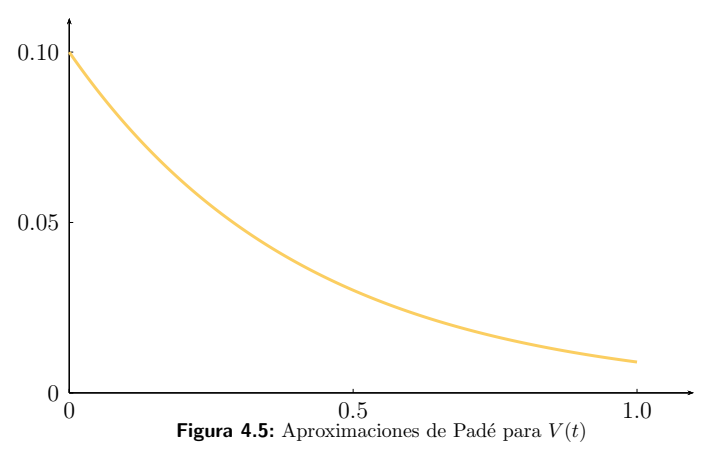

Figure 3: Padé approximants for $V(t)$.

series with respect to the series calculated by other techniques.

Two algorithms were designed to calculate the coefficients of the rational expression corresponding to Padé's approximations of the order $(M, N)$ : the first one was calculated by solving a system of linear equations in which the matrix of coefficients is a Toeplitz matrix, which gave very efficient results numerically; the second algorithm developed is much more efficient than the previous one since it uses properties of continuous fractions in which Padé's approximations correspond to any order under the condition of normality of succession.

Finally, it was verified that the results obtained with the algorithms of this work, gave the same results that those obtained with other methods. Therefore, from the algebraic point of view, the procedure is much simpler and efficient, since the expansion coefficients of the series of $T(t), I(t)$ and $V(t)$ were found in a direct way for the initial model.

\section{Acknowledgments}

The authors gratefully acknowledge the support of the Universidad Tecnológica de Pereira-Colombia, the Group GEDNOL and Research project: Cod. CIE: 3-18-14.

\section{References:}

[1] H. Padé (1892), Sur la représentation approchée d'une fonction par des fractions rationnelles, $\mathrm{An}$ nales scientifiques de l'E.N.S. 3 série, Vol.9, pp. 3-93.

[2] H. Padé (1899). Mémoire sur les développements en fractions continues de la function exponentielle, pouvant servir d'introduction a la théorie des fractions continues algébraiques, Annales scientifiques de l'ENS. 3 série, Vol. 16, pp. 395426.
[3] H. Padé (1907). Recherches sur la convergence des développements en fractions continues d'une certaine catégorie des fonctions, Annales scientifiques de l'ENS, 3 série, Vol. 24, pp. 341-400.

[4] M. Shatnawi (2016). Solving boundary layer problems by residual power series method, Journal of Mathematics Research, Vol. 8, pp. 68-73.

[5] P. Cárdenas (2014). An iterative method for solving two special cases of Lane-Emden type equations. American Journal of Computational Mathematics, Vol. 4, pp. 242-253.

[6] L. Vasquez et al (2014). Direct application of Padé approximant for solving nonlinear differential equations. Springer Plus, Vol. 3, pp. 563-574.

[7] M. Merdan (2007). Homotopy Perturbation Method For Solving a Model For HIV Infection of CD4+ T Cells. Estanbul Ticaret Universitesi Fen Bilimleri Dergisi. pp. 39-52.

[8] E. Celik et al (2003). Arbitrary order numerical method for solving differential algebraic equation by Padé series. Applied Mathematics and Computation, Vol. 137 (1), pp. 57-65.

[9] H. Allouche et al (2017). Numerical solution of singular boundary value problems with logarithmic singularities by padé approximation and collocation methods. J. Comput. Appl. Math, Vol. 311, pp. 324-341.

[10] H. Allouche et al (2019). Highly accurate method for solving singular boundary-value problems via Padé approximation and two-step quartic B-spline collocation. Mediterranean Journal of Math., Vol. 16 (72).

[11] Y. Zong (2019). Simulink-based teaching design for multipath-effect elimination utilizing convolution analysis and pade approximation. Comp. Appl. in Eng. Education, Vol. 27 (3), pp. 631-641.

[12] F. Muhammad et al (2019). Control and simulation impact on nonlinear Hepatitis-B model by using Padé-approximation based Differential Evolution. BioRXiv server for Biology, doi: https://doi.org/10.1101/831636

[13] P. Cárdenas et al (2020). A note on quadratic riccati differential equation using Padé approximant. Int. Journ. of Eng. Tecnh. and Manag. Res., Vol. 7 (6), pp. 117-124. 


\section{Creative Commons Attribution}

\section{License 4.0 (Attribution 4.0}

\section{International, CC BY 4.0)}

This article is published under the terms of the Creative Commons Attribution License 4.0 https://creativecommons.org/licenses/by/4.0/deed.en US 